\title{
Pharmaceutical Activation of Nrf2 Accelerates Diabetic Wound Healing by Exosomes from Bone Marrow Mesenchymal Stem Cells
}

\author{
Lei Wang*, Yuhui Cai*, Qingrong Zhang*, Yi Zhang \\ Department of Burn Rectification, Affiliated Hospital of Nantong University, Nantong, China
}

\begin{abstract}
Background and Objectives: Despite advances in wound treatments, chronic diabetic wounds remain a significant medical challenge. Exosomes from mesenchymal stem cells (MSCs) and small molecule activators of nuclear factor erythroid 2-related factor 2 (Nrf2) have emerged as potential therapies for nonhealing diabetic wounds. This study aimed to evaluate the effects of exosomes from bone marrow-derived MSCs (BMSCs) alone, or in combination with a small molecule activator of Nrf2 on diabetic wound healing.

Methods and Results: BMSCs and endothelial progenitor cells (EPCs) were isolated from the femur and tibia bone marrow of Sprague-Dawley (SD) rats and culture-expanded. Exosomes were harvested from the BMSC culture supernatants through ultracentrifugation. The effects of the exosomes and Nrf2 knockdown, alone or in combination, on EPC tube formation were evaluated. Streptozotocin-induced diabetic rats bearing a fresh full-thickness round wound were treated with the exosomes alone, or in combination with a lentiviral shRNA targeting Nrf2 (Lenti-sh-Nrf2) or tert-butylhydroquinone (tBHQ), a small molecule activator of Nrf2. Two weeks later, wound closure, re-epithelization, collagen deposition, neovascularization, and local inflammation were evaluated. BMSC exosomes promoted while Nrf2 knockdown inhibited EPC tube formation. BMSC exosomes accelerated wound closure, re-epithelization, collagen deposition, and neovascularization, and reduced wound inflammation in diabetic rats. These regenerative and anti-inflammatory effects of the exosomes were inhibited by Lenti-sh-Nrf2 but enhanced by tBHQ administration. Conclusions: BMSC exosomes in combination with a small molecule Nrf2 activator hold promise as a new therapeutic option for chronic diabetic wounds.
\end{abstract}

Keywords: Diabetic wound healing, Bone marrow-derived mesenchymal stem cells, Exosomes, Nrf2, Tert-Butylhydroquinone

Received: April 5, 2021, Revised: August 5, 2021,

Accepted: September 2, 2021, Published online: October 31, 2021

Correspondence to Yi Zhang

Department of Burn Rectification, Affiliated Hospital of Nantong

University, 20 Xisi Road, Nantong 226001, China

Tel: +86-0513-81168901, Fax: +86-0513-81168901

E-mail: zhangyinantong@126.com

*These authors contributed equally to this work.

(c) This is an open-access article distributed under the terms of the Creative Commons Attribution Non-Commercial License (http://creativecommons.org/ licenses/by-nc/4.0/), which permits unrestricted non-commercial use, distribution, and reproduction in any medium, provided the original work is properly cited.

Copyright (c) 2022 by the Korean Society for Stem Cell Research

\section{Introduction}

Chronic nonhealing wounds such as diabetic foot ulcers impact about $15 \%$ of Medicare beneficiaries in the US (1). Nonhealing wounds are associated with low quality of life, and if left untreated, these lesions can lead to severe complications such as gangrene and amputation. In recent years, cell-based therapy has become a promising treatment modality for would healing. Mesenchymal stem cells (MSCs) of various origins such as adipose or bone marrow have been shown to accelerate wound closure by promoting neovascularization and re-epithelization through paracrine signaling (2). Intriguingly, MSC-derived exosomes, 
like their parent cells, can enhance would healing through their immunomodulatory and pro-regenerative properties $(3,4)$. Produced in the endosomal compartment of MSCs, these exosomes contain various cytokines and growth factors such as hepatocyte growth factor (HGF), insulin-like growth factor 1 (IGF1), nerve growth factor (NGF), and stromal cell-derived factor 1 (SDF1) that promote cell migration, cell proliferation, collagen production, and angiogenesis (5). In recent years, researchers have made various efforts to boost the potency of MSC exosomes in accelerating diabetic wound healing, such as preconditioning of their parent cells $(6,7)$ or utilization of hydrogel formulation $(8,9)$.

Impaired wound repair often involves prolonged inflammation and persistently elevated oxidative stress at the wound site. Nuclear factor erythroid 2-related factor 2 (Nrf2) is a transcription factor that regulates the expression of antioxidant proteins including heme oxygenase 1 (HMOX1) and $\mathrm{NAD}(\mathrm{P}) \mathrm{H}$ : quinone oxidoreductase 1 (NQO1). The Nrf2 antioxidant pathway protects cells against oxidative damage caused by external stressors or excessive inflammation (10). Compared with diabetic wildtype mice, the diabetic Nrf2 (-/-) mice showed delayed wound closure with greater oxidative DNA damage and apoptosis (11). The endothelial progenitor cells (EPCs), being able to directly differentiate into endothelial cells, play an important role in revascularization during wound repair $(12,13)$. Of note, Nrf2 overexpression protected diabetic EPCs against oxidative stress-induced dysfunction in vitro (14). Moreover, pharmaceutical activation of $\mathrm{Nrf} 2$ improved EPC functions and accelerated wound closure in diabetic rodents (15-17).

In the present study, we evaluated the effects of bone marrow MSC (BMSC)-derived exosomes alone, or in combination with knockdown or pharmaceutical activation of Nrf2 on would closure, tissue regeneration, and wound inflammation in diabetic rats. The effects of BMSC exosomes and Nrf2 knockdown, alone or in combination, on EPC tube formation in vitro were also investigated.

\section{Materials and Methods}

\section{Animals}

Male Sprague-Dawley (SD) rats (6-8-week-old, 200 250 g) were purchased from Jie Si Jie Lab Animal Ltd. (Shanghai, China). The rats were kept in pathogen-free conditions under the standard $12 \mathrm{~h}$ light/ $12 \mathrm{~h}$ dark cycle. All animal studies were approved by the Ethics Committee of Affiliated Hospital of Nantong University.

\section{Isolation, expansion, and identification of BMSCs}

BMSCs were isolated from the femurs and tibias of SD rats following a previously described procedure (18). The isolated BMSCs were expanded in Dulbecco's Modified Eagle's Medium (DMEM, Gibco) supplemented with 10\% fetal bovine serum (FBS, Gibco) and 1\% penicillin/streptomycin (Invitrogen, USA) at $37^{\circ} \mathrm{C}$ in a humidified air containing $5 \% \mathrm{CO}_{2}$. The medium was changed twice a week. The cells at passage 3 were collected and subjected to analysis on a CytoFleX flow cytometer (Beckman Coulter, USA). The expression of the surface markers CD105, CD90, and CD45 was evaluated with antibodies toward CD105 (MA5-18120; Invitrogen), CD90 (561973; BD Bioscience), and CD45 (554878; BD Bioscience), respectively.

\section{Isolation and identification of BMSC exosomes}

BMSC exosomes were isolated as previously described (19). In brief, BMSCs at passage 3 were cultured overnight until they reached $80 \% \sim 90 \%$ confluence. The medium was changed and the cells were cultured for another 24 hours. The culture supernatant was collected, and the cell debris and apoptotic bodies were removed by low-speed centrifugation. The exosomes were collected by ultracentrifugation at $100,000 \times \mathrm{g}$ and stored at $-80^{\circ} \mathrm{C}$ until further use. Transmission electron microscopy (TEM) was used to inspect freshly isolated exosomes as described previously (19). The expression of the exosome markers CD9, CD63, TSG101 was evaluated with western blot analysis as described in "western blot analysis" in the methods section of this article. The particle size distribution was assessed with dynamic light scattering on a Zetasizer Nano ZS90 analyzer (Malvern Instruments, UK).

\section{Isolation and identification of EPCs}

EPCs were isolated from the femur and tibia bone marrow of SD rats as previously described (20). The isolated EPCs were expanded in Endothelial Cell Growth Basal Medium-2 (EGM-2; Lonza, Switzerland) supplemented with EGM-2 MV SingleQuots (Lonza) and 5\% FBS. The cells at passages 3-8 were used for all studies. The expression of the surface markers CD31, CD34, and CD45 at passage 3 was evaluated on a CytoFleX flow cytometer using antibodies toward CD31 (ab33858; Abcam), CD34 (ab81289; Abcam), and CD45 (554878; BD Bioscience), respectively.

\section{Lentivirus construction and infection}

A short hairpin RNA (shRNA) targeting rat $\mathrm{Nrf} 2$ (sh-Nrf2, 5'-GGTTCAGTGACTCGGAAAT-3') and a con- 
trol shRNA (sh-NC, 5'-CAGATACGACCGCTGAGTT-3') were synthesized and cloned into the pLKO.1 lentiviral vector (HanBio, Shanghai, China) to generate Lenti-sh-Nrf2 and Lenti-sh-NC, respectively. For lentiviral production, the shRNA plasmid, an envelope plasmid, and a packaging plasmid were transfected into 293T cells with Lipofectamine 2000 (Invitrogen). The viral supernatants were collected at 48 hours after transfection, cleared through a $0.45-\mu \mathrm{m}$ filter (EMD Millipore, Billerica, MA), and concentrated by ultracentrifugation. The lentiviral titers of Lenti-sh-Nrf2 and Lenti-sh-NC were $3 \times 10^{8}$ plaque forming units $(\mathrm{pfu}) / \mathrm{ml}$. The EPCs were infected with the concentrated viral supernatants at a multiplicity of infection (MOI) of 10 for 48 hours. After the infection, the cells were selected with puromycinfor stable shRNA expression.

\section{Tube formation assay}

EPCs were seeded at a density of $2 \times 10^{5}$ in Matrigelcoated 24-well plates (BD Biosciences) and incubated for 72 hours at $37^{\circ} \mathrm{C}$. Tube formation in five randomly selected fields was examined under an Olympus light microscope $(\times 400)$. The total tube length was determined.

\section{Rat model of diabetic wound healing}

After one week of acclimation and four weeks on a high-sugar and high-fat diet (21), the rats received a single dose of $35 \mathrm{mg} / \mathrm{kg}$ streptozocin (STZ) (Sigma, St Louis, MO USA) by intraperitoneal injection. A blood glucose test on day 7 after the injection confirmed the induction of diabetes $(>16.7 \mathrm{mmol} / \mathrm{l})$. Under isoflurane anesthesia, a full-thickness round wound with a diameter of $1.5 \mathrm{~cm}$ was created on the shaved dorsal skin. After that, the rats were randomly divided into four groups ( $\mathrm{n}=5$ per group) and immediately received (a) subcutaneous PBS at the wound site, (b) $100 \mu \mathrm{g} / \mathrm{ml}$ subcutaneous BMSC exosomes alone at the wound site, (c) $100 \mu \mathrm{g} / \mathrm{ml}$ subcutaneous BMSC exosomes at the wound site in combination with $200 \mu 1$ intravenous Lenti-sh-Nrf2, (d) $100 \mu \mathrm{g} / \mathrm{ml}$ subcutaneous BMSC exosomes at the wound site in combination with $200 \mu 1$ intravenous Lenti-sh-NC, or (e) $100 \mu \mathrm{g} / \mathrm{ml}$ subcutaneous BMSC-derived exosomes at the wound site in combination with $50 \mathrm{mg} / \mathrm{kg}$ intraperitoneal tert-butylhydroquinone (tBHQ) (Sigma). For exosome administration, the wound was divided into four quadrants, and each quadrant received $50 \mu 1$ injection at the base and edge of the wound. One week later, each group received the same treatment. The wound area was measured on days 0,7 , and 14 after the wounding. The wound size was normalized to that on day $0(100 \%)$. The rats were euthan- ized on day 14, and the wound tissues were collected for histological, immunohistochemical, ELISA, and western blot analysis.

\section{Histological analysis}

The wound tissues were fixed in $10 \%$ formalin, dehydrated in a series of graded-alcohol, embedded in paraffin, and cut into $4-\mu \mathrm{m}$ sections. The extent of healing was evaluated with hematoxylin and eosin (H\&E) and Masson's trichrome (MT) staining.

\section{Western blot analysis}

The exosomes, cell lysates, and tissues homogenates (40 $\mu \mathrm{g}$ of total protein) were subjected to SDS-PAGE and transferred to nitrocellulose membranes (Millipore). After blocking in 5\% nonfat milk for 1 hour, the membranes were incubated with primary antibodies toward CD9 (1 : 500; MA5-31980; Invitrogen), CD63 (1 : 5,000; PA5-100713; Invitrogen), TSG101 ( $1: 1,000$; ab133586; Abcam), Nrf2 ( $1: 1,000 ;$ ab89443; Abcam), and $\beta$-actin (1:1,000; ab8226; Abcam), respectively, overnight at $4^{\circ} \mathrm{C}$. The membranes were subsequently incubated with horseradish peroxidase (HRP)-conjugated secondary antibodies for 1 hour at room temperature. Protein bands were detected using an ECL detection system (GE Healthcare, USA) and quantified by densitometric analysis with the ImageJ software (National Institutes of Health, USA).

\section{Immunohistochemistry}

The expression of CD31 in the wound tissues was evaluated using immunohistochemical staining with an anti-CD31 antibody (1:2,000; ab182981; Abcam) as previously described (22). Immunoreactivity signals from at least five random fields were recorded, and the CD31 expression level was calculated as the average integrated optical density (IOD) per area.

\section{ELISAs}

To evaluate the level of wound inflammation, the wound tissues were homogenized with a rotor-stator homogenizer (TissueRuptor, Qiagen, Germantown, MD) in cold PBS. The resulting homogenates were centrifuged at $12,000 \mathrm{rpm}$ for $10 \mathrm{~min}$ at $4^{\circ} \mathrm{C}$. The supernatants were collected, and the protein concentrations were determined with a BCA protein assay kit (Beyotime, Shanghai, China). The concentrations of TNF- $\alpha$, IL-1 $\beta$, IL-4, and IL-10 were determined using ELISA kits from eBioscience (San Diego, CA, USA) following manufacturer's instructions. 


\section{Statistical analysis}

All data were analyzed with the SPSS 21.0 statistical software and subjected to the normal distribution and variance homogeneity tests. All data are presented as mean \pm standard deviation. The unpaired $t$ test was used to compare normally distributed data between two groups. Comparisons among multiple groups were performed with the one-way analysis of variance (ANOVA) with Tukey's post hoc test. A p values of less than 0.05 was deemed statistically significant.

\section{Results}

\section{BMSC exosomes were successfully harvested and identified}

To prepare BMSC exosomes, we first isolated BMSCs from the femurs and tibias of SD rats and expanded the cells in DMEM supplemented with 10\% FBS. MSCs are typically identified by the coexpression of CD105 and CD90 and negative expression of CD45 (23). As indicated by the flow cytometric analysis, a vast majority of the cell population at passage 3 showed expression of CD105 (97.3\%) and CD90 (95.8\%) and negative expression of CD45 (0.33\%) (Fig. 1A), confirming their BMSC identity. Next, we harvested exosomes from the culture supernatants of passage-3 BMSCs through ultracentrifugation. Similar to previous reports (24), these exosomes exhibited a typical spherical double-membrane structure under TEM, with a median size of around $100 \mathrm{~nm}$ (Fig. 1B and 1C). Moreover, the expression of the exosome markers CD9, CD63, TSG101 was confirmed with western blot analysis (Fig. 1D).

\section{EPCs were successfully isolated and identified}

To evaluate in vitro tube formation, we isolated EPCs from the femur and tibia bone marrow of SD rats, and expanded the cells in EGM-2 supplemented with EGM-2 MV SingleQuots and 5\% FBS. Late EPCs have a high proliferative capacity and can form vascular tubes in Matrigel in vitro. These cells are typically identified by the coexpression of CD31 and CD34 and negative expression of CD45 (25). As indicated by the flow cytometric analysis, a vast majority of passage-3 cells showed positive expression of CD31 (98.5\%) and CD34 (96.8\%), but negative expression of CD45 (1.27\%) (Fig. 2A), confirming that the cells we obtained were late EPCs suitable for the study of in vitro tube formation.

\section{Nrf2 knockdown inhibited EPC tube formation promoted by BMSC exosomes}

Next, we evaluated the effects of BMSC exosomes and Nrf2 knockdown, alone or in combination, on EPC tube formation in vitro. To evaluate the effects of Nrf2 knockdown, we generated EPCs stably expressing sh-Nrf2. Nrf2 knockdown was confirmed with western blot analysis (Fig. 2B). After 72-hour incubation in Matrigel-coated plates, EPCs formed microscopic tubular-like networks (Fig. 2C). Compared with control, the cells treated with BMSC exosomes showed better tube formation as indicated in a significantly greater total tube length (Fig. 2D). We found that Nrf2 knockdown led to impaired EPC tube formation on Matrigel. In addition, Nrf2 knockdown inhibited tube formation promoted by BMSC exosomes (Fig. 2C and 2D).
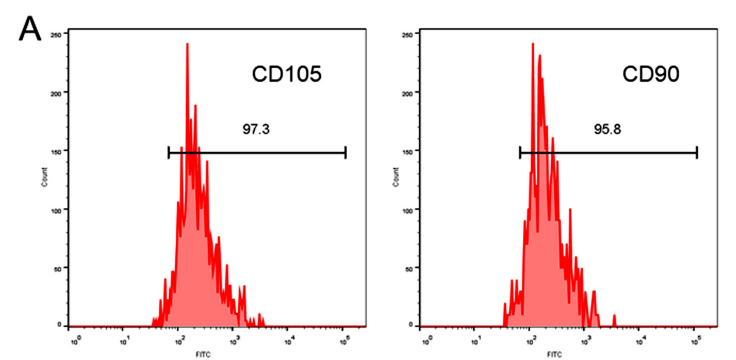

B

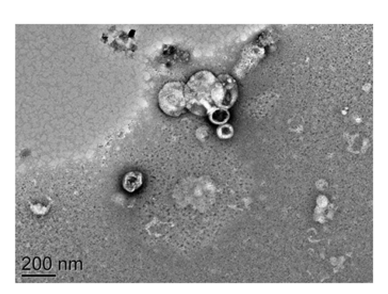

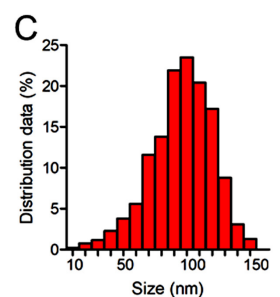
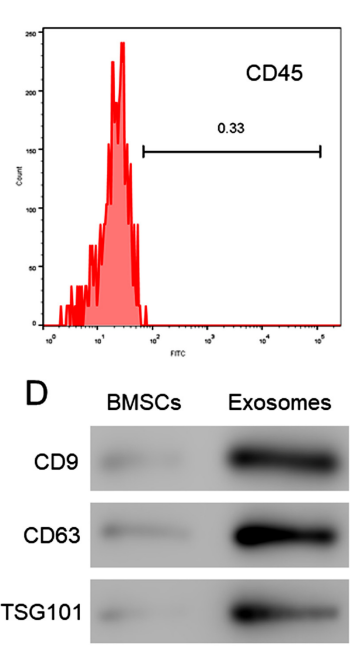

Fig. 1. Identification of BMSCs and BMSC exosomes. (A) The detection of the surface markers CD105, CD90, and CD45 in isolated BMSCs with flow cytometry. (B) A TEM image of BMSC exosomes. Scale bar $=100 \mathrm{~nm}$. (C) The particle size distribution of BMSC exosomes determined with light scattering. (D) The detection of the exosome markers CD9, CD63, and TSG101 in BMSC exosomes with western blot analysis. 
A
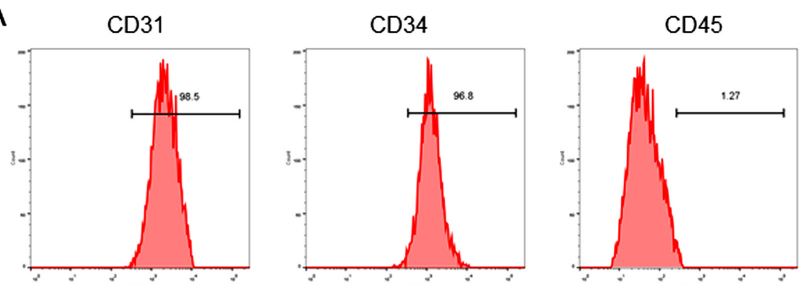

B

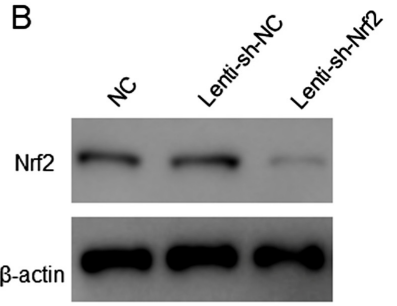

C

NC
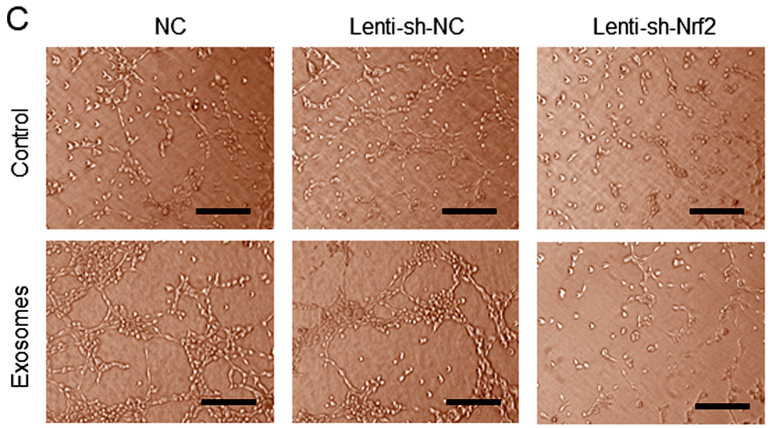

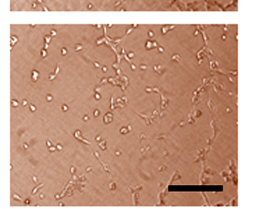

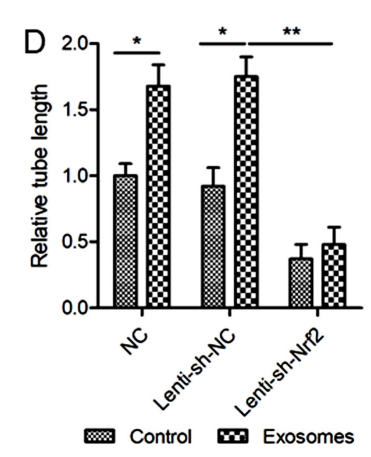

Fig. 2. The effects of BMSC exosomes and Nrf2 knockdown, alone or in combination, on EPC tube formation. (A) The detection of the surface markers CD31, CD34, and CD45 in isolated EPCs with flow cytometry. (B) EPCs were transfected with Lenti-sh-Nrf2 or Lenti-sh-NC for 48 hours. The Nrf2 protein levels were determined with western blot analysis. (C, D) EPCs stably expressing sh-Nrf2 or sh-NC were treated with BMSC exosomes $(200 \mu \mathrm{g} / \mathrm{ml})$ for 72 hours. (C) Tube formation was observed under a microscope. Scale bar $=100 \mu \mathrm{m}$. (D) The relative tube length in each treatment group was determined. $\mathrm{N}=3, * \mathrm{p}<0.05,{ }^{* *} \mathrm{p}<$ 0.01 .

A
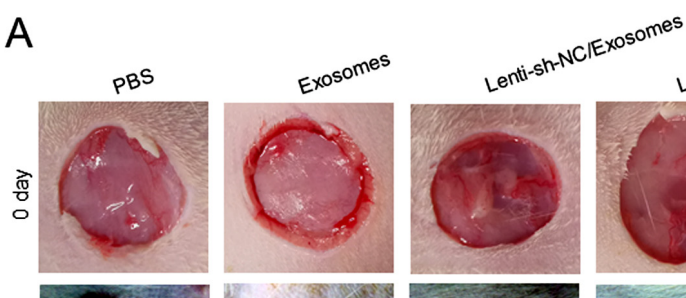

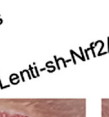

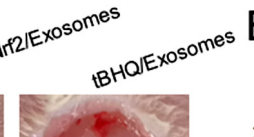

B
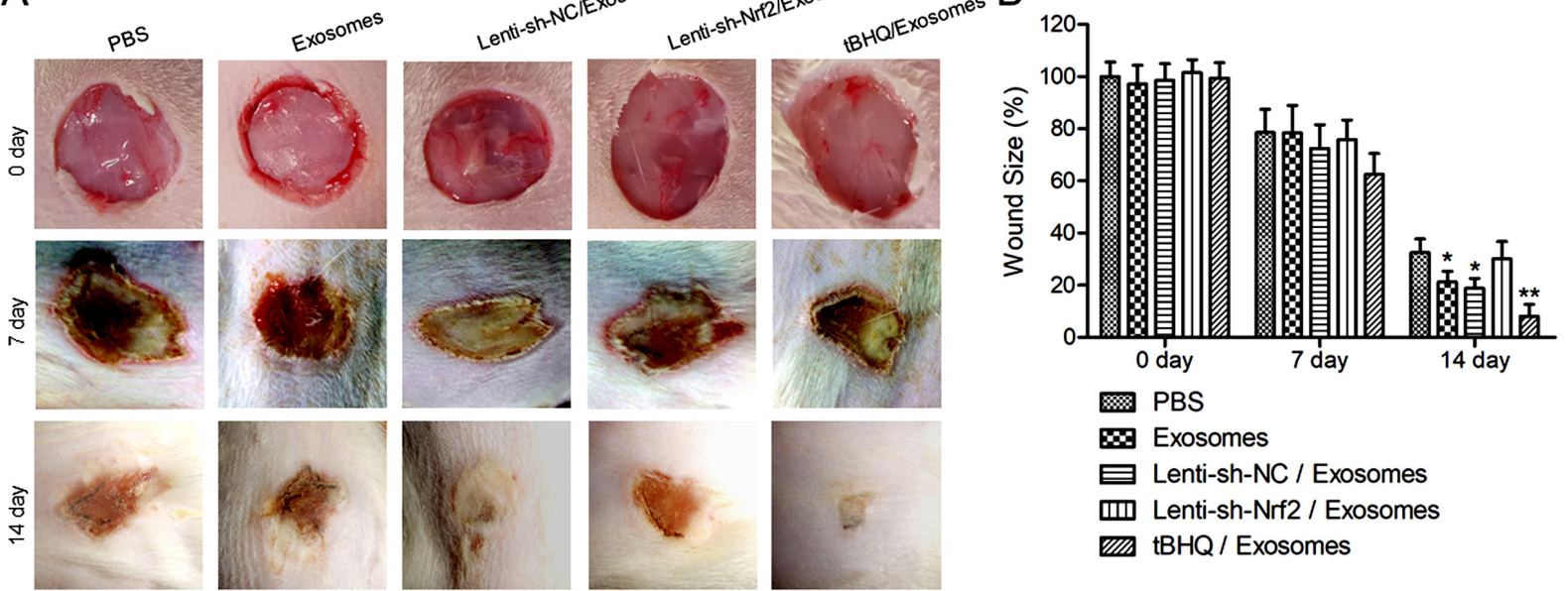

Fig. 3. The effects of BMSC exosomes alone, or in combination with knockdown or pharmaceutical activation of Nrf2 on wound closure in diabetic rats. STZ-induced diabetic rats received $100 \mu \mathrm{g} / \mathrm{ml}$ subcutaneous BMSC exosomes alone at the wound site, or in combination with $200 \mu \mathrm{l}$ intravenous Lenti-sh-Nrf2 or Lenti-sh-NC, or $50 \mathrm{mg} / \mathrm{kg}$ intraperitoneal tBHQ immediately after the wounding and again a week after. Rats that received PBS were included as control. (A) The photo images of the wounds at day 0,7 , and 14 after the wounding. (B) The wound size at day 0,7 , and 14 after the wounding. $\mathrm{N}=5 ;{ }^{*} \mathrm{p}<0.05,{ }^{*} \mathrm{p}<0.01$ vs. PBS control.

\section{Pharmaceutical activation of Nrf2 enhanced wound healing in diabetic rats by BMSC exosomes}

In our rat model of diabetic wound healing, local subcutaneous injection of BMSC exosomes significantly accelerated wound closure at day 14 after the wounding (Fig. $3 \mathrm{~A}$ and $3 \mathrm{~B}$ ). It has been shown that $\mathrm{tBHQ}$ activates Nrf2 by binding to the Keap1 protein and interfering with Keap1-mediated Nrf2 degradation (26). The exosome-treated wounds showed increased Nrf2 expression. Indeed, wounds treated with both exosomes and $\mathrm{BHHQ}$ showed a sig- nificantly higher level of the Nrf2 protein than those treated with exosomes alone (Fig. 4A). Compared with control, the exosome-treated wounds exhibited enhanced re-epithelization, collagen deposition, and neovascularization as indicated in H\&E staining (Fig. 4B), Masson staining (Fig. 4C), and CD31 immunohistochemistry (Fig. 5), respectively. Of note, the exosome-treated wounds also showed reduced inflammation, as they exhibited decreased levels of the inflammatory cytokines TNF- $\alpha$ and IL-1 $\beta$ (Fig. 6A and $6 \mathrm{~B})$ and increased levels of the anti-inflammatory cyto- 

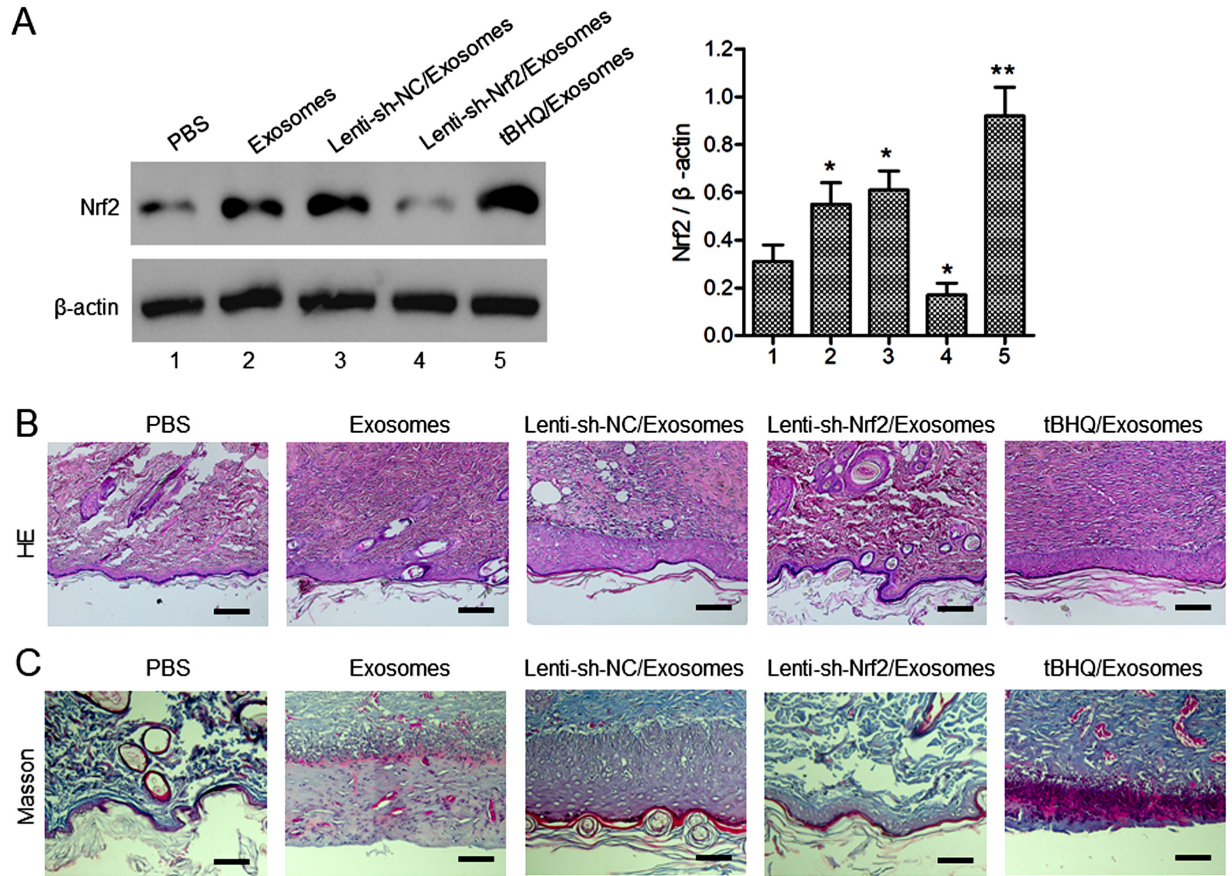

tBHQ/Exosomes

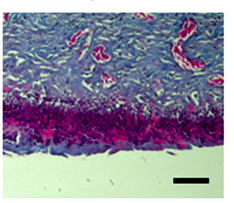

Fig. 4. The effects of BMSC exosomes alone, or in combination with knockdown or pharmaceutical activation of Nrf2 on wound tissue regeneration in diabetic rats. (A) The Nrf2 protein levels in the wound tissues at day 14 after the wounding were evaluated with western blot analysis. The STZ-induced diabetic rats were treated after skin wounding as described in Fig. 3. (A, B) Wound re-epithelization and collagen deposition were evaluated with H\&E (B) and Masson (C) staining of the wound tissues at day 14 after the wounding. Scale $\mathrm{bar}=100 \mu \mathrm{m}$. $\mathrm{N}=5 ; * \mathrm{p}<0.05, * * \mathrm{p}<0.01$ vs. PBS control.
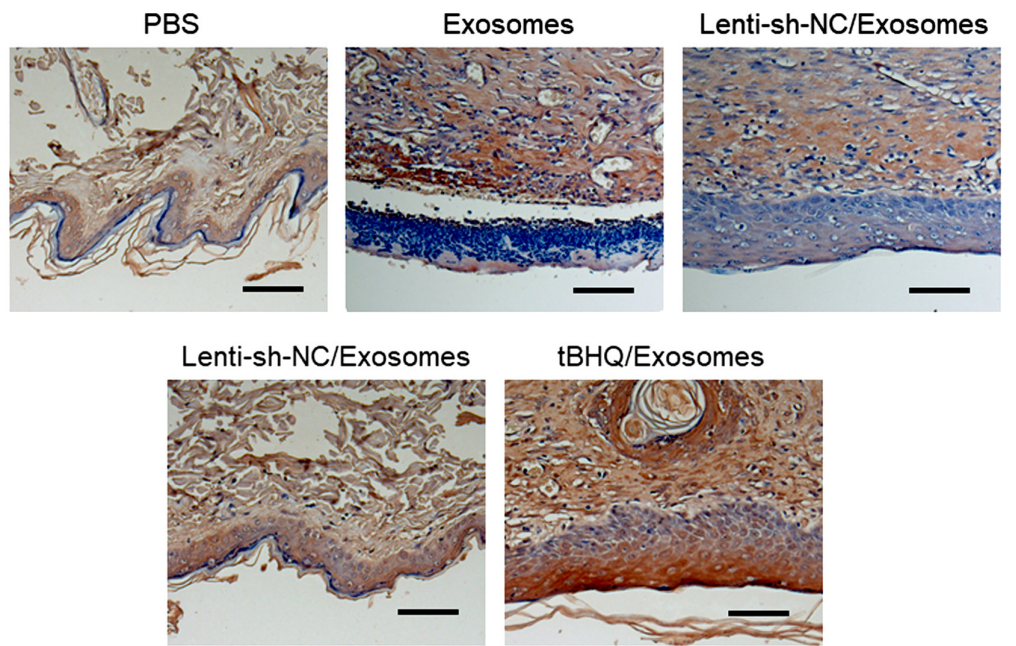

tBHQ/Exosomes

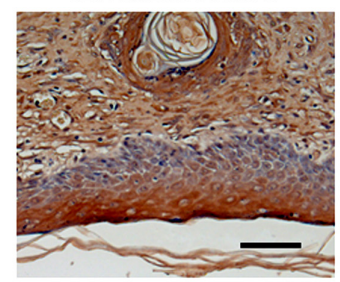

Fig. 5. The effects of BMSC exosomes alone, or in combination with knockdown or pharmaceutical activation of Nrf2 on wound neovascularization in diabetic rats. The STZ-induced diabetic rats were treated after skin wounding as described in Fig. 3 . The CD31 expression in the wound tissues at day 14 after the wounding was detected with immunohistochemistry. Scale bar=100 $\mu \mathrm{m} . \mathrm{N}=5 ;{ }^{*} \mathrm{p}<0.05,{ }^{* *} \mathrm{p}$ $<0.01$ vs. PBS control.

kines IL-4 and IL-10 (Fig. 6C and 6D). These regenerative and anti-inflammatory effects of the exosomes were eliminated by Lenti-sh-Nrf2 administration, suggesting that the exosomes promoted wound healing at least partially through activation of the Nrf2 anti-oxidant pathway. Finally, BMSC exosomes in combination with the Nrf2 activator $\mathrm{tBHQ}$ showed greater efficacy in accelerating wound healing and suppressing inflammation than exo- somes alone.

\section{Discussion}

Chronic diabetic wounds represent a substantial socioeconomic burden for the healthcare system worldwide. Although treatment strategies have greatly improved over the past decades, there remains a significant need for ad- 

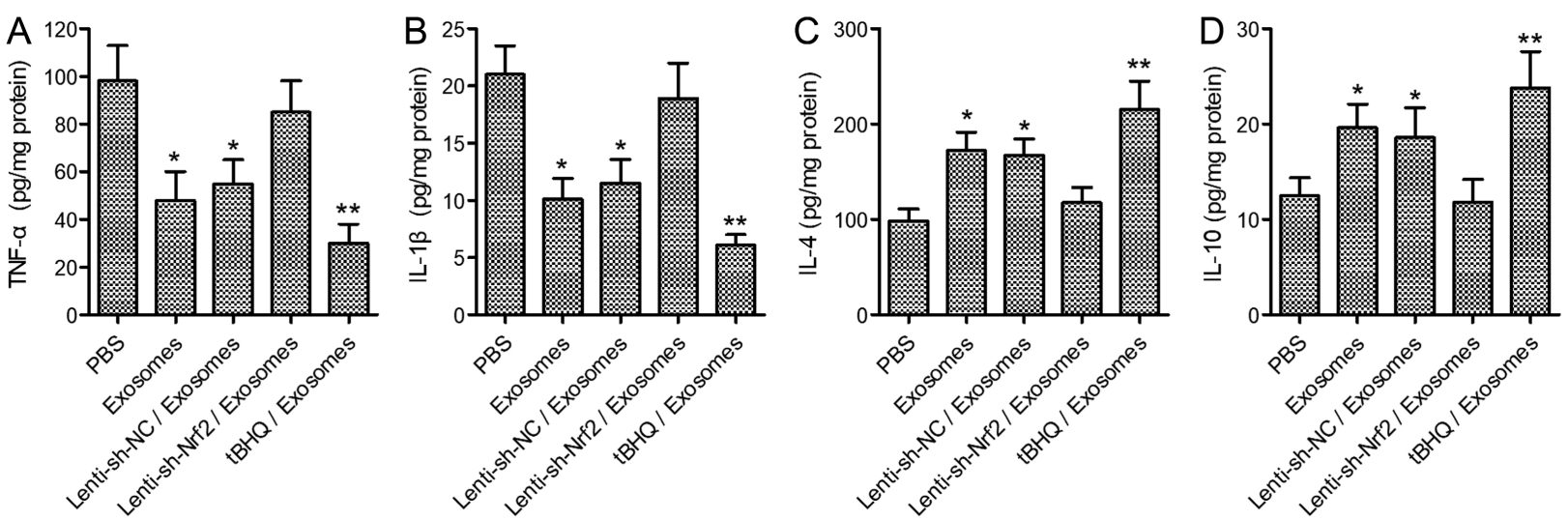

Fig. 6. The effects of BMSC exosomes alone, or in combination with knockdown or pharmaceutical activation of Nrf2 on wound inflammation in diabetic rats. The STZ-induced diabetic rats were treated after skin wounding as described in Fig. 3. The levels of TNF- $\alpha$, $\mathrm{IL}-1 \beta, \mathrm{IL}-4$, and IL-10 in the wound tissues at day 14 after the wounding were determined with ELISA. N=5; ${ }^{*} \mathrm{p}<0.05, * * \mathrm{p}<0.01$ vs. PBS control.

ditional therapeutic options (27). In this study, we found that BMSC exosomes promoted EPC tube formation in vitro and accelerated healing and inhibited inflammation of diabetic wounds in vivo. These therapeutic benefits of the exosomes in vivo were enhanced by pharmaceutical activation of $\mathrm{Nrf2}$, indicating that exosomes in combination with a Nrf2 activator may hold promise as a novel therapeutic option for chronic diabetic wounds.

Given the ease to isolate and expand MSCs from readily accessible tissue sources, MSCs have been the most studied cell therapy platform in regenerative medicine. However, the outcomes of several advanced clinical trials have fallen short of expectations (28), prompting the researchers to move toward cell-free approaches to avoid the difficult challenges of cell-based therapy such as immune rejection. Accumulating evidence has indicated that MSCs enhance cell migration, angiogenesis, and re-epithelialization during wound repair through paracrine signals mediated by secretomes $(29,30)$. Consequently, exosomes derived from MSCs of various origins have emerged as a promising acellular therapy for wound healing, with BMSCs and umbilical cord MSCs (UCMSCs) showing the greatest regenerative efficacy (5). Hyperglycemia-induced chronic inflammation is a critical contributing factor to impaired wound healing in diabetic patients. Diabetic wounds have elevated levels of inflammatory cytokines such as TNF- $\alpha$ and IL- $1 \beta$, which promote the pro-inflammatory macrophage Ml phenotype (31). Studies have shown that MSC-derived exosomes can reduce diabetic wound inflammation by promoting macrophage M2 polarization (32). In this study, BMSC exosomes upregulated Nrf2 and downregulated TNF- $\alpha$ and IL-1 $\beta$ in diabetic wounds, supporting that the "healing power" of the exosomes is at least partially mediated by its anti-oxidant and anti-inflammatory properties. Following this line of thought, it is reasonable to speculate that MSC exosomes in combination with an anti-oxidant or anti-inflammatory agent would be more efficacious than exosomes alone in healing diabetic wounds.

In recently years, researchers have tried various methods to boost the efficacy of MSC exosomes in healing diabetic wounds. These methods often involve preconditioning of the parent MSCs such as stimulation with deferoxamine, $\mathrm{Fe}_{3} \mathrm{O}_{4}$ nanoparticles, melatonin, or atorvastatin $(6$, $7,32,33)$. In particular, exosomes from melatonin-stimulated MSCs accelerated diabetic wound healing by promoting macrophage $\mathrm{M} 2$ polarization and suppressing inflammatory cytokines (32). In this study, we boosted the efficacy of BMSC-derived exosomes by pharmaceutical activation of the Nrf2 anti-oxidant pathway. A 2018 report showed that exosomes from adipose-derived MSCs (ADMSCs) overexpressing Nrf2 enhanced healing of diabetic foot ulcers (34). Compared with wildtype exosomes, the Nrf2overexpressing exosomes exhibited greater efficacy in accelerating diabetic wound repair while reducing the levels of inflammation and oxidative stress-related proteins (34). Compared with their genetic approach, our method of using a small molecule to activate $\mathrm{Nrf2}$ is probably more suitable for potential clinical application.

Biomaterial-based hydrogels that structurally imitate the natural extracellular matrix (ECM) have been successfully exploited as a drug delivery system for wound treatments (35). In particular, MSC exosomes loaded in a bioactive hydrogel have achieved better outcomes in healing 
diabetic wounds than exosomes or hydrogel alone $(8,9)$. We speculate that hydrogel-formulated exosomes in combination with a Nrf2 activator may achieve even greater efficacy in treating chronic diabetic wounds. Further studies are warranted.

In conclusion, we demonstrated that BMSC exosomes in combination with pharmaceutical activation of $\mathrm{Nrf} 2$ may hold promise as an effective treatment option for diabetic wounds.

\section{Acknowledgments}

The project is funded by the Nantong Technology Program (NO.MS12017006-6).

\section{Potential Conflict of Interest}

The authors have no conflicting financial interest.

\section{Author Contributions}

Lei Wang, Yuhui Cai and Qingrong Zhang contributed to the conception of the study; Lei Wang, Yuhui Cai and Qingrong Zhang performed the experiments; Lei Wang, Yuhui Cai and Qingrong Zhang contributed significantly to data analysis and manuscript preparation; Lei Wang, Yuhui Cai and Qingrong Zhang perform the analysis with constructive discussions; Yi Zhang supervised and checked the manuscript.

\section{References}

1. Nussbaum SR, Carter MJ, Fife CE, DaVanzo J, Haught R, Nusgart M, Cartwright D. An economic evaluation of the impact, cost, and medicare policy implications of chronic nonhealing wounds. Value Health 2018;21:27-32

2. Kosaric N, Kiwanuka H, Gurtner GC. Stem cell therapies for wound healing. Expert Opin Biol Ther 2019;19:575-585

3. Hettich BF, Ben-Yehuda Greenwald M, Werner S, Leroux JC. Exosomes for wound healing: purification optimization and identification of bioactive components. Adv Sci (Weinh) 2020;7:2002596

4. Silva AM, Teixeira JH, Almeida MI, Gonçalves RM, Barbosa MA, Santos SG. Extracellular vesicles: immunomodulatory messengers in the context of tissue repair/regeneration. Eur J Pharm Sci 2017;98:86-95

5. Hoang DH, Nguyen TD, Nguyen HP, Nguyen XH, Do PTX, Dang VD, Dam PTM, Bui HTH, Trinh MQ Vu DM, Hoang NTM, Thanh LN, Than UTT. Differential wound healing capacity of mesenchymal stem cell-derived exosomes originated from bone marrow, adipose tissue and umbilical cord under serum- and xeno-free condition. Front Mol Biosci 2020;7:119

6. Ding J, Wang X, Chen B, Zhang J, Xu J. Exosomes derived from human bone marrow mesenchymal stem cells stimu- lated by deferoxamine accelerate cutaneous wound healing by promoting angiogenesis. Biomed Res Int 2019;2019: 9742765

7. Wu D, Kang L, Tian J, Wu Y, Liu J, Li Z, Wu X, Huang Y, Gao B, Wang H, Wu Z, Qiu G. Exosomes derived from bone mesenchymal stem cells with the stimulation of $\mathrm{Fe}_{3} \mathrm{O}_{4}$ nanoparticles and static magnetic field enhance wound healing through upregulated miR-21-5p. Int J Nanomedicine 2020;15:7979-7993

8. Wang C, Liang C, Wang R, Yao X, Guo P, Yuan W, Liu Y, Song Y, Li Z, Xie X. The fabrication of a highly efficient self-healing hydrogel from natural biopolymers loaded with exosomes for the synergistic promotion of severe wound healing. Biomater Sci 2019;8:313-324

9. Wang $\mathrm{C}$, Wang $\mathrm{M}, \mathrm{Xu} \mathrm{T}$, Zhang $\mathrm{X}$, Lin $\mathrm{C}$, Gao W, Xu H, Lei B, Mao C. Engineering bioactive self-healing antibacterial exosomes hydrogel for promoting chronic diabetic wound healing and complete skin regeneration. Theranostics 2019;9:65-76

10. He F, Ru X, Wen T. NRF2, a transcription factor for stress response and beyond. Int J Mol Sci 2020;21:4777

11. Long $M$, Rojo de la Vega $M$, Wen Q, Bharara $M$, Jiang T, Zhang R, Zhou S, Wong PK, Wondrak GT, Zheng H, Zhang DD. An essential role of NRF2 in diabetic wound healing. Diabetes 2016;65:780-793

12. Murasawa S, Asahara T. Endothelial progenitor cells for vasculogenesis. Physiology (Bethesda) 2005;20:36-42

13. Kaushik K, Das A. Endothelial progenitor cell therapy for chronic wound tissue regeneration. Cytotherapy 2019;21: $1137-1150$

14. Wang RY, Liu LH, Liu H, Wu KF, An J, Wang Q, Liu Y, Bai LJ, Qi BM, Qi BL, Zhang L. Nrf2 protects against diabetic dysfunction of endothelial progenitor cells via regulating cell senescence. Int J Mol Med 2018;42:1327-1340

15. Fan J, Liu H, Wang J, Zeng J, Tan Y, Wang Y, Yu X, Li W, Wang P, Yang Z, Dai X. Procyanidin B2 improves endothelial progenitor cell function and promotes wound healing in diabetic mice via activating Nrf2. J Cell Mol Med 2021;25:652-665

16. Sun X, Wang X, Zhao Z, Chen J, Li C, Zhao G. Paeoniflorin accelerates foot wound healing in diabetic rats though activating the Nrf2 pathway. Acta Histochem 2020;122:151649

17. Li Y, Ma F, Li H, Song Y, Zhang H, Jiang Z, Wu H. Dimethyl fumarate accelerates wound healing under diabetic condition. J Mol Endocrinol 2018;61:163-172

18. Gnecchi M, Melo LG. Bone marrow-derived mesenchymal stem cells: isolation, expansion, characterization, viral transduction, and production of conditioned medium. Methods Mol Biol 2009;482:281-294

19. Chen Y, Ding H, Wei M, Zha W, Guan S, Liu N, Li Y, Tan Y, Wang Y, Wu F. MSC-secreted exosomal H19 promotes trophoblast cell invasion and migration by downregulating let-7b and upregulating FOXO1. Mol Ther Nucleic Acids 2020;19:1237-1249

20. Zhang J, Zhang H, Chen Y, Fu J, Lei Y, Sun J, Tang B. Platelet-derived growth factor D promotes the angiogenic 
capacity of endothelial progenitor cells. Mol Med Rep 2019; 19:125-132

21. Wang L, Wang F, Zhao L, Yang W, Wan X, Yue C, Mo Z. Mesenchymal stem cells coated by the extracellular matrix promote wound healing in diabetic rats. Stem Cells Int 2019;2019:9564869

22. Zhao P, Sui BD, Liu N, Lv YJ, Zheng CX, Lu YB, Huang WT, Zhou CH, Chen J, Pang DL, Fei DD, Xuan K, Hu $\mathrm{CH}$, Jin Y. Anti-aging pharmacology in cutaneous wound healing: effects of metformin, resveratrol, and rapamycin by local application. Aging Cell 2017;16:1083-1093

23. Mendicino M, Bailey AM, Wonnacott K, Puri RK, Bauer SR. MSC-based product characterization for clinical trials: an FDA perspective. Cell Stem Cell 2014;14:141-145

24. Shi Y, Kang X, Wang Y, Bian X, He G, Zhou M, Tang $\mathrm{K}$. Exosomes derived from bone marrow stromal cells (BMSCs) enhance tendon-bone healing by regulating macrophage polarization. Med Sci Monit 2020;26:e923328

25. Werling NJ, Thorpe R, Zhao Y. A systematic approach to the establishment and characterization of endothelial progenitor cells for gene therapy. Hum Gene Ther Methods 2013;24:171-184

26. Li W, Kong AN. Molecular mechanisms of Nrf2-mediated antioxidant response. Mol Carcinog 2009;48:91-104

27. Rekha PD, Rao SS, Sahana TG, Prabhu A. Diabetic wound management. Br J Community Nurs 2018;23(Sup9):S16-S22

28. Galipeau J, Sensébé L. Mesenchymal stromal cells: clinical challenges and therapeutic opportunities. Cell Stem Cell 2018;22:824-833
29. Yoon BS, Moon JH, Jun EK, Kim J, Maeng I, Kim JS, Lee JH, Baik CS, Kim A, Cho KS, Lee JH, Lee HH, Whang KY, You S. Secretory profiles and wound healing effects of human amniotic fluid-derived mesenchymal stem cells. Stem Cells Dev 2010;19:887-902

30. Cao Y, Gang X, Sun C, Wang G. Mesenchymal stem cells improve healing of diabetic foot ulcer. J Diabetes Res 2017;2017:9328347

31. Fui LW, Lok MPW, Govindasamy V, Yong TK, Lek TK, Das AK. Understanding the multifaceted mechanisms of diabetic wound healing and therapeutic application of stem cells conditioned medium in the healing process. J Tissue Eng Regen Med 2019;13:2218-2233

32. Liu W, Yu M, Xie D, Wang L, Ye C, Zhu Q, Liu F, Yang L. Melatonin-stimulated MSC-derived exosomes improve diabetic wound healing through regulating macrophage M1 and M2 polarization by targeting the PTEN/AKT pathway. Stem Cell Res Ther 2020;11:259

33. Yu M, Liu W, Li J, Lu J, Lu H, Jia W, Liu F. Exosomes derived from atorvastatin-pretreated MSC accelerate diabetic wound repair by enhancing angiogenesis via AKT/ eNOS pathway. Stem Cell Res Ther 2020;11:350

34. Li X, Xie X, Lian W, Shi R, Han S, Zhang H, Lu L, Li M. Exosomes from adipose-derived stem cells overexpressing Nrf2 accelerate cutaneous wound healing by promoting vascularization in a diabetic foot ulcer rat model. Exp Mol Med 2018;50:1-14

35. Sharifzadeh G, Hosseinkhani H. Biomolecule-responsive hydrogels in medicine. Adv Healthc Mater 2017;6:1700801 